J. Lake Sci. (湖泊科学), 2015, 27(2): 343-351

http: //www. jlakes. org. E-mail : jlakes@niglas.ac.cn

(C) 2015 by Journal of Lake Sciences

\title{
鄱阳湖夏季水面蒸发与蒸发血蒸发的比较”
}

\author{
赵晓松 ${ }^{1}$, 李 梅 $^{2}$, 王仕刚 ${ }^{3}$, 刘元波 ${ }^{1 * *}$ \\ ( $1:$ 中国科学院南京地理与湖泊研究所湖泊与环境国家重点实验室,南京 210008) \\ $(2$ : 江西省水文局,南昌 330002$)$ \\ ( 3 : 江西省鄱阳湖水文局,九江 332800$)$
}

\begin{abstract}
摘 要: 水面蒸发是湖泊水量平衡要素的重要组成部分. 基于传统蒸发血观测蒸发不能代表实际水面蒸发, 而实际水面 蒸发特征仍不清楚. 本研究基于浴度相关系统观测的鄱阳湖水体实际水面蒸发过程, 在小时和日尺度分析了水面蒸发的 变化规律及其主要影响因子, 并与蒸发血蒸发进行比较. 研究表明, 实际水面蒸发日变化波动剧烈, 变化范围在 $0 \sim$ $0.4 \mathrm{~mm} / \mathrm{h}$ 之间. 水面蒸发的日变化特征主要受风速的影响. 鄱阳湖 8 月份日水面蒸发量与蒸发血蒸发量在总体趋势上具 有很好的一致性. 8 月份平均日水面蒸发速率 $(5.90 \mathrm{~mm} / \mathrm{d})$ 比蒸发血蒸发速率 $(5.65 \mathrm{~mm} / \mathrm{d})$ 高 $4.6 \%$. 水面日蒸发量与蒸 发血蒸发量的比值在 8 月上、中、下旬平均值分别为 $1.24 、 1.00 、 0.92$, 呈现下降的趋势. 鄱阳湖夏季水面日蒸发量与风速 和相对湿度相关性显著, 而蒸发血蒸发与净辐射、气温、饱和水汽压差和相对湿度均呈显著相关. 这是由于蒸发血水体容 积小, 与湖泊相比其水体热存储能力小, 因此更容易受到环境因子的影响.
\end{abstract}

关键词: 实际水面蒸发;蒸发血蒸发;涡度相关;鄱阳湖

\section{Comparison of actual water evaporation and pan evaporation in summer over the Lake Poyang, China}

\author{
ZHAO Xiaosong ${ }^{1}$, LI Mei ${ }^{2}$, WANG Shigang ${ }^{3} \&$ LIU Yuanbo ${ }^{1}$ \\ (1: State Key Laboratory of Lake Science and Environment, Nanjing Institute of Geography and Limnology, Chinese Academy \\ of Sciences, Nanjing 210008, P. R. China) \\ (2: Hydrological Bureau of Jiangxi Province, Nanchang 330002, P. R. China) \\ (3: Hydrological Bureau of Poyang Lake, Jiangxi Province, Jiujiang 332800, P. R. China)
}

\begin{abstract}
Evaporation is an important factor in water balance of lakes. The evaporation pan is a traditional method to observe water evaporation, while it cannot represent actual water evaporation. So the characters of actual water evaporation are still unclear. In this study, the actual water evaporation was measured by an eddy covariance system over the Lake Poyang in August of 2013 . We analyze the diurnal and monthly variations of water evaporation and its main influencing factors, and compare it with pan evaporation measured at Tangyin station. The results show that the water evaporation has strong turbulence in diurnal variations in the range of $0-0.4 \mathrm{~mm} / \mathrm{h}$. The diurnal variation of water evaporation is mainly controlled by the wind speed. The daily water evaporation has similar trend with daily pan evaporation in August over the Lake Poyang. The average water evaporation in August is 5.90 $\mathrm{mm} / \mathrm{d}$, which is higher than $4.6 \%$ for average pan evaporation $(5.65 \mathrm{~mm} / \mathrm{d})$. The ratio of water evaporation to pan evaporation in August shows decreasing trend, and the ten-day average ration was $1.24,1.00,0.92$, respectively. The daily water evaporation in August has good relationship with wind speed and relative humidity, and pan evaporation was significantly related to net radiation, air temperature, the saturation vapor pressure deficit and relative humidity. This is due to evaporation pan has small volume and weak water heat storage capacity comparing with the lake, thus it easily affected by environmental factors.
\end{abstract}

Keywords: Actual water evaporation; pan evaporation; eddy covariance; Lake Poyang

* 国家重点基础研究发展计划“973”项目(2012CB417003)、国家自然科学基金项目(41471022)、中国科学院南京地理 与湖泊研究所“一三五” 战略发展规划项目 ( NIGLAS2012135001) 和江西省水利厅科技项目 (KT201121) 联合资助. 2014-03-18 收稿;2014-08-18 收修改稿. 赵晓松(1980 ), 女, 博士, 助理研究员; E-mail:xszhao@ niglas. ac. cn.

** 通信作者;E-mail:ybliu@ niglas. ac. cn. 
水面蒸发是影响江河湖泊、水库等自然水体的水量循环和能量平衡的重要因素. 全球陆地表面的蒸发 量约占陆地降水量的 $60 \% \sim 65 \%{ }^{[1]}$. 开展水面蒸发研究对于认识区域气候、旱涝变化趋势, 水资源形成及变 化规律, 水资源评价等具有重要的意义. 目前, 国内外对蒸散发进行计算时, 多基于蒸发器 (血) (如 E-601 型 蒸发器、 $\varphi 20 \mathrm{~cm}$ 蒸发血) 观测数据 ${ }^{[2]}$. 蒸发器 ( III.) 由于本身及周围空气的动力和热力条件与流域地表有所 不同, 测得的蒸发量并不能代表自然界真实的蒸发量, 有时偏差非常大, 并且不同类型仪器的比较也很困 难. 即使通过蒸发血折算系数的校正也不能很好地反映蒸发量的动态变化, 因为蒸发血折算系数受多种因 素影响而且随季节变化 ${ }^{[3-4]}$. 尽管蒸发血观测的蒸发量并不能代表真实水体蒸发, 但其长期观测对认识潜在 蒸发的变化规律仍具有积极的理论价值.

随着全球地表温度升高影响的加剧, 气候变化预测的研究认为气温升高将增加陆地水体的蒸发量, 然 而观测的结果却发现蒸发血蒸发长期变化呈降低的趋势 ${ }^{[5-6]}$. 这一现象称为蒸发悖论, 这一争论的焦点是关 于自然水体的实际蒸发机制仍不清楚. 目前,涡度相关系统作为地表水热通量观测的国际标准手段,已被广 泛应用于地表植被和内陆水体中 ${ }^{[7-8]}$. 国际上利用涡度相关系统进行的湖泊水体通量观测, 最早报道见于 1991 年, 在印度尼西亚的 Toba 湖 $^{[9]}$ 和美国浅水湖泊 ${ }^{[10]}$ 进行了数日至数月不等的短期通量观测. 而长期的 湖泊水体观测始于 21 世纪初, 分别在加拿大的 Great Slave 湖 ${ }^{[11-12]}$ 和 Great Bear 湖 ${ }^{[13]}$ 和美国 Ross Barnett 水 库 ${ }^{[14]}$. 美国的 Williams 湖 ${ }^{[15]}$ 、以色列的 Eshkol 水库、智利北部 Tilopozo 湿地 ${ }^{[16]}$ 和芬兰南部的 Valkea-Kotinen 湖 ${ }^{[17]}$ 等也开展了短期测量. 多数研究基于长期或短期的观测, 分析了不同大小、不同纬度湖泊或水库等水面 蒸发的特征及在不同时间尺度水面蒸发的影响因子 ${ }^{[17-18]}$.

我国学者关于水面蒸发观测和估算早期开展了大量研究, 主要集中在 3 个方面: (1) 应用不同蒸发器 观测的水面蒸发折算系数的研究 ${ }^{[8,19-22]}$. 研究表明,折算系数受到辐射、水温、风速和储热量等时空差异的影 响 ${ }^{[8,23]}$, 长江流域基于 E601 蒸发血对 $20 \mathrm{~m}^{2}$ 蒸发池之间的折算系数在 $0.75 \sim 1.09$ 之间变化 ${ }^{[22]}$; (2) 水面蒸 发模型的研究 ${ }^{[24-29]}$. 基于彭曼公式和道尔顿公式建立了全国通用的水面蒸发公式 ${ }^{[26,28]}$; (3) 水面蒸发量变 化趋势的研究 ${ }^{[4,30-33]}$. 基于蒸发皿蒸发分析了全国水面蒸发特征 ${ }^{[30]}$, 以及长江流域 ${ }^{[33]}$ 和鄱阳湖流域 ${ }^{[32,34]}$ 蒸 发特征及其影响因子. 国内基于浴度相关方法观测湖泊水体水热通量变化过程的研究才刚刚开始, 例如太 湖, 而其主要关注内容是大气与湖泊间的水热交换系数 ${ }^{[35]}$. 基于浴度相关直接观测湖泊水体蒸发的研究还 未展开. 作为我国的最大淡水湖泊, 鄱阳湖最大水面面积达到 4000 余 $\mathrm{km}^{2}$. 长期以来, 鄱阳湖的水面蒸发量 基本上依据蒸发皿蒸发量进行计算 ${ }^{[32]}$. 实际水面蒸发过程及主要影响因素不明, 这也导致目前该湖的湖泊 水量平衡计算中存在较大的不确定性.

本研究基于浴度相关系统观测鄱阳湖水体的水面蒸发, 分析不同时间尺度鄱阳湖夏季水面蒸发的变化 特征, 比较夏季水面蒸发与蒸发血蒸发在变化趋势上的不同以及环境因子对两者影响机制的差异, 为准确 估算湖泊水面蒸发的长期变化奠定基础.

\section{1 研究方法}

\section{1 研究站点与观测仪器}

鄱阳湖位于 $28^{\circ} 30^{\prime} \sim 30^{\circ} \mathrm{N}, 115^{\circ} 30^{\prime} \sim 117^{\circ} \mathrm{E}$ ( 图 1), 本研究的试验站点位于鄱阳湖东部, 都昌县附近的 蛇山岛上 $\left(29^{\circ} 05^{\prime} \mathrm{N}, 116^{\circ} 24^{\prime} \mathrm{E}\right.$ ). 鄱阳湖面积为 $4380 \mathrm{~km}^{2}$ (不包括军山湖, 2010 年鄱阳湖基础地理信息测量成 果), 年内水位变化剧烈, 平均变幅达 $11 \mathrm{~m}$ 左右 ${ }^{[36]}$. 鄱阳湖地属亚热带湿润季风气候, 湖区年平均气温为 $17.1^{\circ} \mathrm{C}$, 多年平均降水量为 $1570 \mathrm{~mm}$, 降水集中在 4-6 月, 占全年降水的 $45 \% \sim 50 \%$. 主风向为北和北偏西 风. 西北方向距离棠荫岛约 $2 \mathrm{~km}$, 距离城镇边界的岸边约 $6 \mathrm{~km}$. 蛇山岛面积约为 $0.2 \mathrm{~km}^{2}$, 南北长 $500 \mathrm{~m}$, 东 西长 $450 \mathrm{~m}$. 棠荫岛面积约为 $0.8 \mathrm{~km}^{2}$.

为减少蛇山岛本身对观测的影响, 观测铁搭架设在蛇山岛北部, 浴度相关系统仪器安装在 $38 \mathrm{~m}$ 的观测 铁搭上. 考虑到岛本身的高度, 观测高度距离湖面 $58 \mathrm{~m}$. 采用三维超声风速和 $\mathrm{CO}_{2} / \mathrm{H}_{2} \mathrm{O}$ 分析仪 (EC150, Campbell Scientific Inc., Logan, UT, USA) 测量三维风速和大气的 $\mathrm{H}_{2} \mathrm{O}$ 浓度, 采用频率为 $10 \mathrm{~Hz}$, 通过数据采 集器 (CR3000, Campbell Inc., ) 进行存储. 用净辐射仪 (CNR4, Kipp \& Zonen B. V., Delft, The Netherlands) 观测辐射平衡的 4 个组分 (向下、向上长波辐射, 向下、向上短波辐射). 采用小气候系统仪器观测气温和相 
对湿度 (HMP155A, Vaisala Helsinki, Fenland). 涡度相关系统和小气候系统安装高度为 $38 \mathrm{~m}$,辐射观测安装 高度为 $2.5 \mathrm{~m}$. 水热通量、辐射和小气候系统数据的输出步长为 $30 \mathrm{~min}$. 用水温计 (SWL1-1) 测量 $50 \mathrm{~cm}$ 水深 处的水温. 应用 E601-B 蒸发血测量蒸发,地点位于棠荫岛气象观测站内. 水温和蒸发血蒸发的采样频率为 每天一次,于上午 $8: 00$ 采集数据. 浴度相关系统仪器和小气候仪器于 2013 年 7 月 26 日安装并开始观测. 本 文选取 2013 年 8 月 1 日- 8 月 30 日数据进行分析,避免水位降低后洲滩出露的影响,通量信息主要来自于 鄱阳湖水体. 测量期间风向以北偏西及南偏东为主.

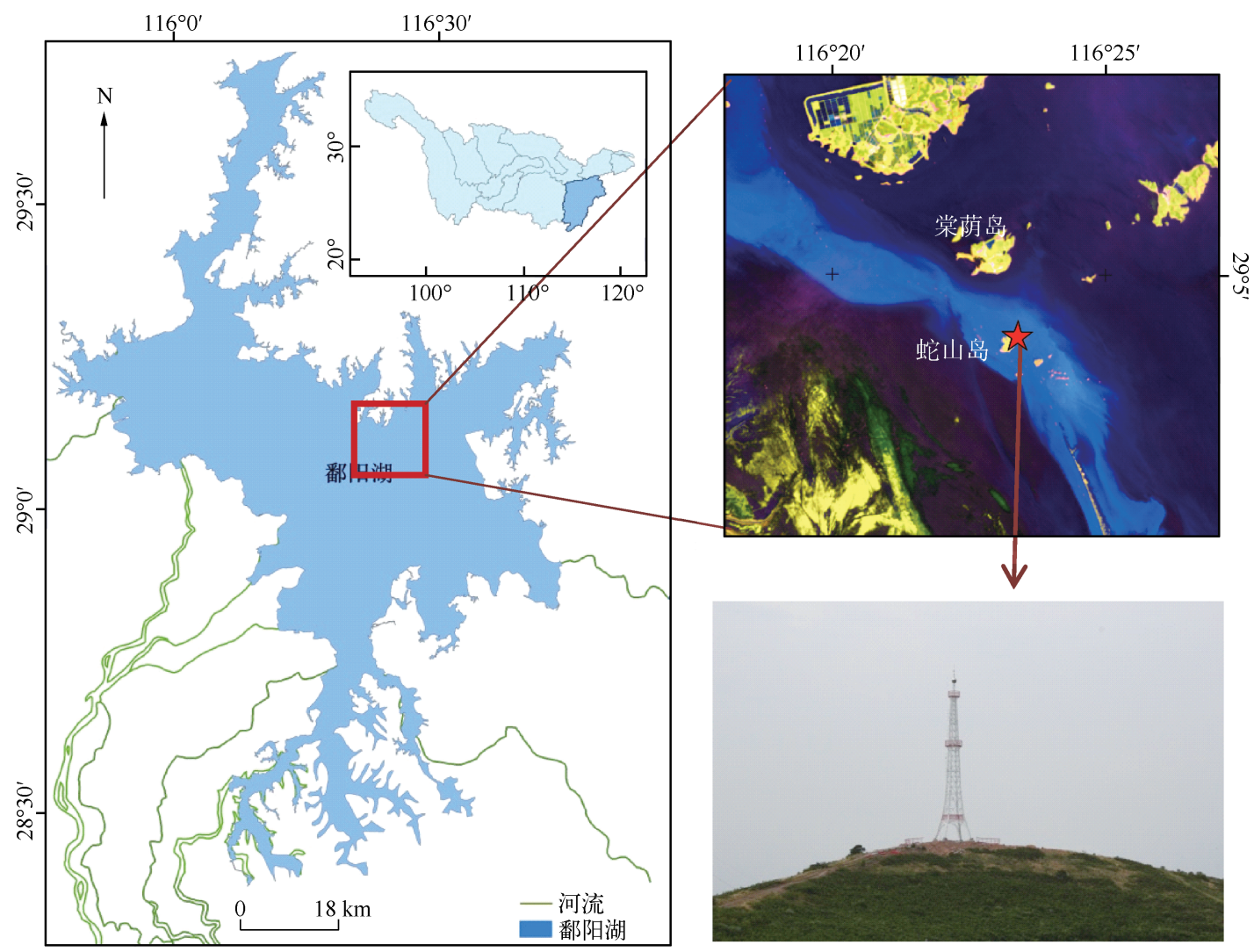

图 1 鄱阳湖及蛇山岛观测点位置

Fig. 1 The map of the Lake Poyang and measurement site at Sheshan Island

\section{2 数据处理过程}

对涡度相关观测的 $10 \mathrm{~Hz}$ 数据进行篮选和修正. 数据处理采用目前普遍使用较为成熟的方法, 应用 $\mathrm{Ed}-$ dyPro 软件进行处理, 包括: (1) 数据篮选, 4 倍标准差剔除野点, 信号强度和诊断值判断数据有效性; (2) 应 用平面坐标拟合方法进行倾斜修正 ${ }^{[37]}$; (3) 频率损失修正 ${ }^{[38]}$; (4) 感热的超声虚温修正 ${ }^{[39]}$; (5) 密度效应 修正: WPL 修正 ${ }^{[40]}$. 通过湍流谱分析和平稳性检验和总体湍流特征检验进行数据质量控制 ${ }^{[41-42]}$. 获得 3 个 评价等级的通量数据, 分别为 0 (高质量)、1 (中质量) 、2(低质量) 数据.

浴度相关技术是通过快速测定大气的物理量 (如温度, 水汽密度, $\mathrm{CO}_{2}$ 浓度等) 与垂直风速的协方差来 计算湍流通量的一种方法. 通过测量水汽密度可以获得水汽通量 (即潜热通量 $L E$ ) :

$$
L E=\lambda \overline{w^{\prime} \rho_{\mathrm{V}}{ }^{\prime}}
$$

式中, $w$ 为垂直风速, $\rho_{\mathrm{V}}$ 为水汽密度 $\mathrm{kg} / \mathrm{m}^{3}, \overline{w^{\prime} \rho_{\mathrm{V}}{ }^{\prime}}$ 为垂直风速和水汽密度脉动协方差. $\lambda$ 为汽化潜热 $(2440 \mathrm{~J} / \mathrm{kg})$. 在分析中,水面蒸发 $(\mathrm{E})$ 单位通常用 $\mathrm{mm} / \mathrm{h}$ 或 $\mathrm{mm} / \mathrm{d}$ 表示. 水面蒸发可通过潜热通量转换, 公式为 $E=$ 
$L E / \lambda$, 此时 $E$ 的单位为 $\mathrm{kg} /\left(\mathrm{m}^{2} \cdot \mathrm{s}\right)$, 根据水的密度 $\left(\mathrm{kg} / \mathrm{m}^{3}\right)$, 再乘以相应的时间步长, 即可转换为 $\mathrm{mm} / \mathrm{h}$ 或 $\mathrm{mm} / \mathrm{d}$ 的蒸发速率单位.

进行数据处理和评价后, 输出 $30 \mathrm{~min}$ 评价的通量数据, 用于进一步分析. 在进行环境因子等分析时, 需 要剔除数据质量为 2 的数据. 在计算日蒸散量时, 需要对缺失的数据进行插补, 本研究所选时段数据缺失比 例为 $9.5 \%$, 当连续缺失数据小于 3 小时, 应用线性内插方法进行数据插补 ${ }^{[43]}$.

\section{3 通量观测源区分析}

浴度相关系统观测的通量代表了上风向一定范围内的通量信息, 即通量源区 (Footprint). 对于不均匀的 下垫面条件, 常需利用 Footprint 分析了解下垫面不同位置对传感器所测湍流通量贡献的大小, 及对观测数 据做质量判别. 关于通量源区的计算有很多解析模型 ${ }^{[44-46]}$. 本研究应用 Hsieh 等 $^{[44]}$ 的方法分析不同大气条 件下 Footprint 分布, 来确定观测时段内通量信息来源. 通量源区分布如图 2 所示. 8 月 1 日-8 月 30 日期间 风向主要来自西北和东南方向. 在稳定大气条件下, $90 \%$ 的通量信息来自于上风向距观测塔 $5 \mathrm{~km}$ 范围内. 在 不稳定大气条件下, 通量源区的范围较小, 在距观测塔 $1 \sim 2 \mathrm{~km}$ 范围内. 当风向为北和西北方向时, 且在稳定 条件下, 水热通量的源区受到棠荫岛的影响, 但棠荫岛的面积仅占该方向通量源区面积的 4\%. 若考虑到棠 荫岛所处位置的通量贡献率( 小于 $20 \%$ ), 则棠荫岛对水面蒸发的影响小于 $1 \%$. 当风向为南和东南方向时， 水热通量的源区受到蛇山岛本身的影响,而蛇山岛的面积仅占该方向通量源区面积的 $1 \%$,即使是在不稳定 条件通量源区范围较小时, 蛇山岛的面积占通量源区面积比例仍小于 $3 \%$. 若考虑到蛇山岛所处位置的通量 贡献率 (小于 $40 \%$ ), 则蛇山岛对水面蒸发的影响约为 $1 \%$. 总体而言, 观测期内主要通量信息均来自于水 体,受蛇山岛和棠荫岛的影响较小, 可以忽略.
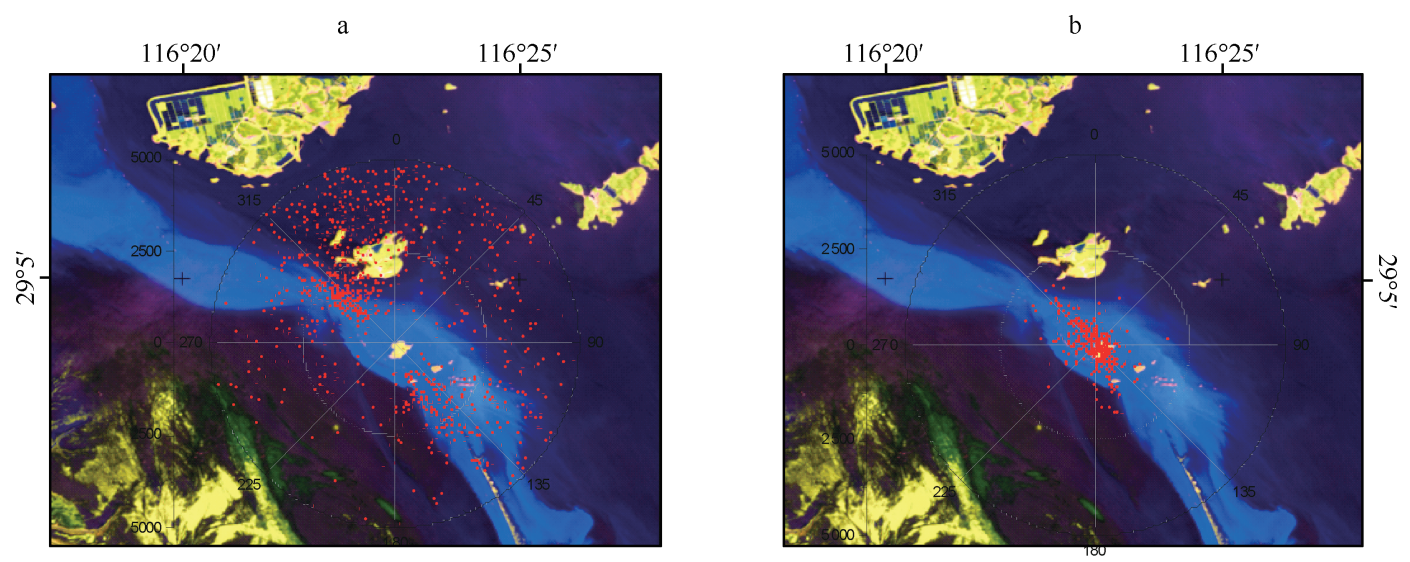

图 2 源区 Footprint 分布图: 稳定条件 (a)、不稳定条件 (b) $\left(0^{\circ}\right.$ 表示北向, $90^{\circ}$ 表示东向, $180^{\circ}$ 表示南向, $270^{\circ}$ 表示西向) (底图为 Landsat 8 6-5-4 波段合成影像, 时间为 2013 年 8 月 2 日, 水位 $15.6 \mathrm{~m}$ )

Fig. 2 The distribution of footprint for energy fluxes observation around the Sheshan Island under stable atmospheric condition(a) and unstable atmospheric condition(b)

\section{2 研究结果}

\section{1 水面蒸发的日变化特征}

通过浴度相关测量的 $30 \mathrm{~min}$ 平均潜热通量转换为水面蒸发速率, 选取 8月 13-15 日为代表, 分析水面 蒸发速率和主要环境因子的日变化过程 (图 3). 根据净辐射的分布特征, 选取的时间段内, 8 月 13 日为典型 的晴天过程, 净辐射呈单峰正态分布, 最大值达 $600 \mathrm{~W} / \mathrm{m}^{2}$, 出现在正午 $12: 00$ 左右, 而 8 月 14 日和 15 日为 多云或阴天的天气, 净辐射呈波动变化, 其日平均值低于晴天. 水面蒸发速率日变化过程较净辐射呈现更为 剧烈的波动变化, 且无明显的日变化规律. 水面蒸发速率在 $0 \sim 0.4 \mathrm{~mm} / \mathrm{h}$ 之间变化, 在夜间有零星的负值出 现. 水面蒸发速率的波动变化与净辐射、气温和饱和水汽压差 (VPD) 的日变化过程无显著相关, 而与风速的 
波动具有很好的一致性. 统计分析显 示, 日变化过程中水面蒸发速率与风 速的 Pearson 相关系数为 $0.27(P=$ $0.001)$. 当风速较大时往往对应较高 的水面蒸发速率. 这说明在日尺度水 面蒸发速率主要受风速的影响, 风速 加强了大气与水面之间的湍流交换 作用, 从而促进水面蒸发速率的升 高 ${ }^{[16-17]}$. 尽管风速是水面蒸发速率变 化的主要影响因子, 气温和饱和水汽 压差也对水面蒸发有抑制或促进作 用 ${ }^{[17]}$. 例如 8 月 14 日的日变化过程, 在 5:00-10:00 之间风速较大, 对应 较高的水面蒸发速率, 10:00 之后风 速迅速下降,而此时气温和饱和水汽 压差升高, 促进了水面蒸发过程, 使 得水面仍保持较高的蒸发速率. 当 20:00 之后风速再次升高, 对应较低 的气温和 VPD 值, 此时水面蒸发速率 也较低.

\section{2 实际水面蒸发与蒸发血蒸发的 比较}

基于浴度相关系统是目前普遍 认可的最可靠的实际蒸发观测方 法 ${ }^{[7]}$, 为研究自然水体蒸发变化规律 提供了直接的证据. 与蒸发血观测的 蒸发量进行比较, 可以检验蒸发血蒸
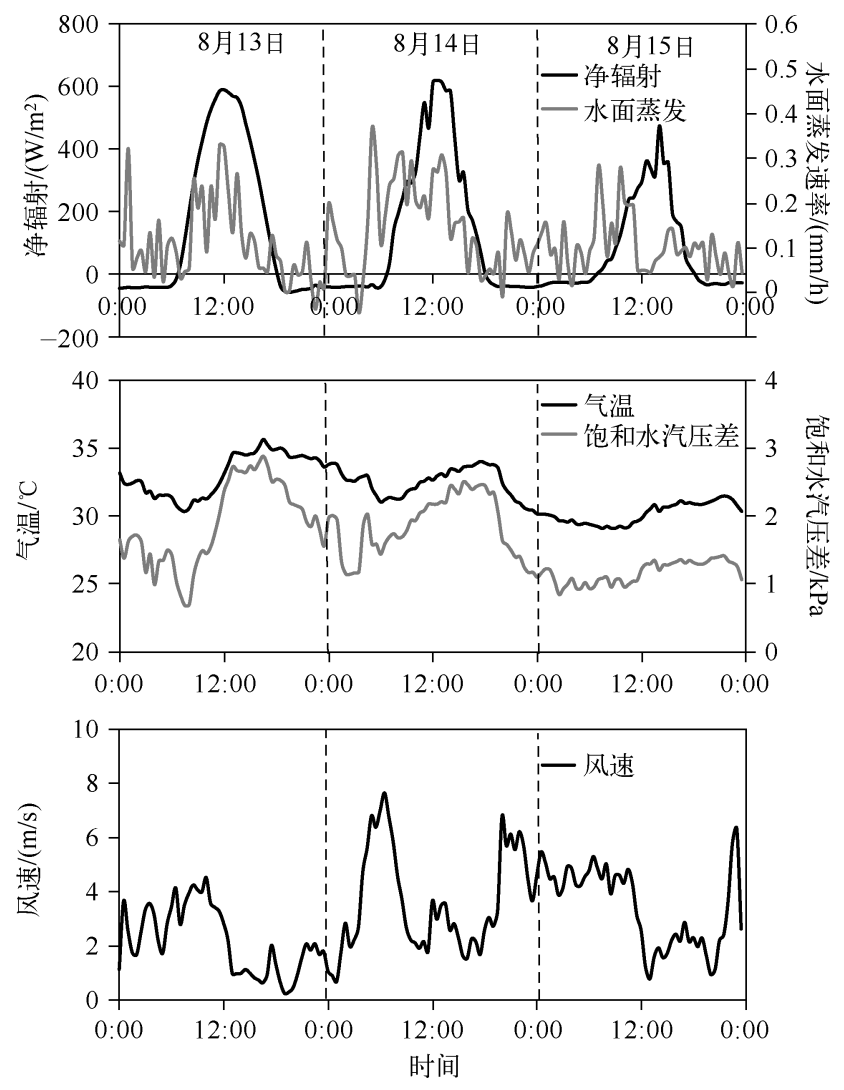

图 3 鄱阳湖水热通量及辐射、气象要素日变化过程 (8月 13 日一 8 月 15 日)

Fig. 3 Diurnal variation of energy fluxes, net radiation and meteorological factors over the Lake Poyang from Aug. 13 to Aug. 15 发的代表性和准确性. 8 月份蒸发血.

日蒸发量与浴度相关观测的水面蒸发量, 及其他环境因子的变化如图 4 所示. 由于蒸发血蒸发是上午 $8: 00$ 观测, 其蒸发量代表前日 $8: 00$ 至当日 8:00 的蒸发量. 为了与其进行对比, 涡度相关观测的水面蒸发采用前 日 8:00 至当日 8:00 的蒸发量累计作为当日的蒸发量. 从时间序列图中可以看出 (图 4a), 两者在一定程度 上表现出一致性,在 8 月初蒸发量较大,然后呈逐渐降低的趋势. 两者均呈现波动变化, 在相位上略有差异, 峰值和谷值不完全同步. 总体来看, 8 月份日均净辐射、气温和饱和水汽压差均呈下降趋势. 平均风速的变化 波动较大, 总体呈下降趋势, 但在 8 月中下旬出现较大风速. 在 8 月初水面蒸发速率明显高于蒸发血蒸发速 率, 而 8 月 25 日- 8 月 28 日显著低于蒸发血蒸发速率. 蒸发血蒸发速率平均值为 $5.65 \mathrm{~mm} / \mathrm{d}$, 标准差为 $1.18 \mathrm{~mm} / \mathrm{d}$, 而涡度相关的水面蒸发速率平均值为 $5.90 \mathrm{~mm} / \mathrm{d}$, 标准差为 $2.20 \mathrm{~mm} / \mathrm{d}$. 标准差大说明实际蒸发 量存在更大的波动范围.

散点比较分析表明 (图 5), 水面蒸发速率与蒸发血蒸发速率两者具有很好的一致性,但观测点较为分散. 涡度相关水面蒸发速率略大于蒸发血蒸发速率, 斜率为 0.3 , 相关系数为 0.31 , 均方根误差 $(R M S E)$ 为 $1.86 \mathrm{~mm} / \mathrm{d} .8$ 月累计蒸发血蒸发量为 $175.0 \mathrm{~mm}$, 而涡度相关观测的水面蒸发量为 $183.04 \mathrm{~mm}$, 较前者高 $4.6 \%$. 闵骞等 ${ }^{[34]}$ 基于蒸发血折算系数计算鄱阳湖水面蒸发, 1955-2004 年平均年蒸发量为 $1081.2 \mathrm{~mm}$, 鄱 阳湖年均蒸发量为 $1081.2 \mathrm{~mm}$ (1955-2004 年), 其中 8 月平均蒸发量为 $169.5 \mathrm{~mm}$, 占全年的 $15.7 \%$, 这一 数值低于本研究 8 月蒸发量. 而对应 1955-2004 年多年平均 8 月气温为 $28.96^{\circ} \mathrm{C}$, 也低于本研究中 2013 年 8 月的月均气温 $\left(30.9^{\circ} \mathrm{C}\right)$. 鄱阳湖夏季 8 月份实际水面日蒸发量 $\left(E_{\mathrm{EC}}\right)$ 与蒸发血目蒸发量 $\left(E_{\mathrm{pan}}\right)$ 的比值 

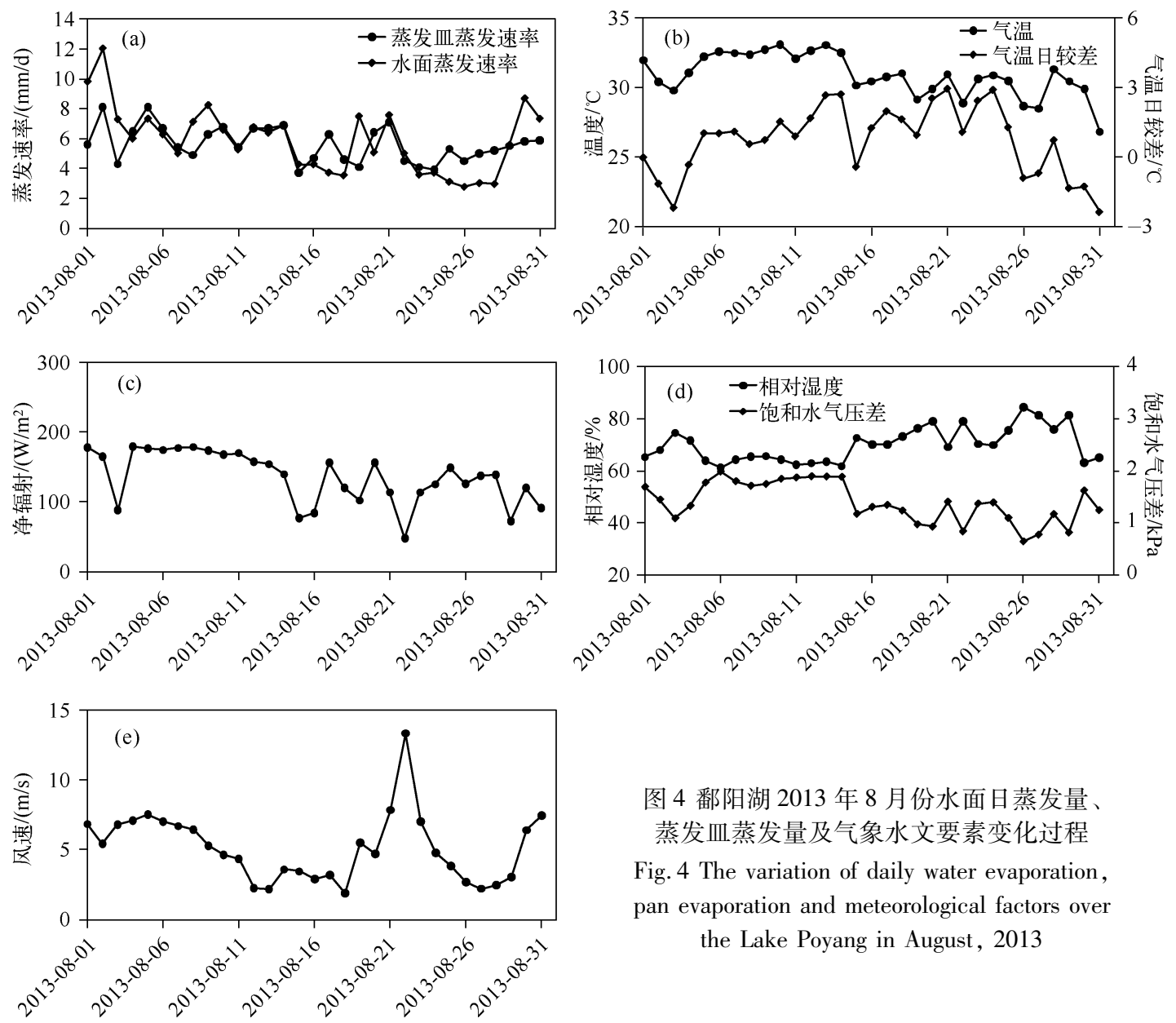

图 4 鄱阳湖 2013 年 8 月份水面日蒸发量、 蒸发皿蒸发量及气象水文要素变化过程

Fig. 4 The variation of daily water evaporation, pan evaporation and meteorological factors over the Lake Poyang in August, 2013

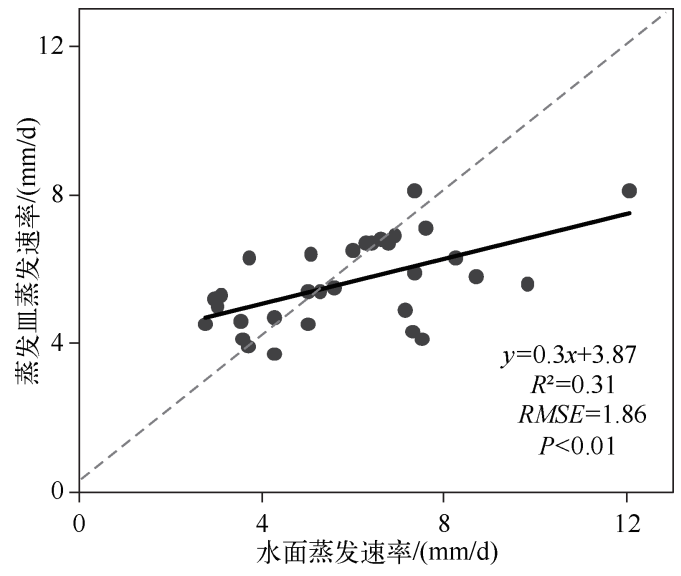

图 5 鄱阳湖 2013 年 8 月份蒸发血蒸发与涡度相关蒸发对比

Fig. 5 The comparison of pan evaporation and water evaporation measured by eddy covariance over the Lake Poyang in August, 2013

$\left(E_{\mathrm{EC}} / E_{\mathrm{pan}}\right.$ ) 在 $0.5 \sim 1.7$ 之间变化 (图 6). 计算 $E_{\mathrm{EC}} / E_{\mathrm{pan}} 10$ 天平均值得到, 8 月上旬 $E_{\mathrm{EC}} / E_{\mathrm{pan}}$ 平均为 1.24 , 中旬为 1.00 , 下旬为 0.92. 闵骞 ${ }^{[31]}$ 分析了鄱阳湖都昌蒸发站 $\mathrm{E} 601$ 蒸发血对漂浮水面蒸发器的折算系 数,发现折算系数在 6-10 月大于 1 , 其他 月份小于 1 . 研究表明,这一比值受到不同 地点和不同季节的影响 ${ }^{[47]}$. 在干旱的以色 列地区,标准 Class-A 蒸发血观测的水库蒸 发量与浴度相关蒸发量的比值在 0.96 1.94 之间变化 $(7-9$ 月), 表现为蒸发血蒸 发高于水面蒸发 ${ }^{[47]}$. 在 Sparkling 湖, 蒸发 皿蒸发与湖泊蒸发存在明显的季节变化差 异. 两者在 7 月和 8 月有很好的一致性,在 9-10 月水面蒸发比蒸发血蒸发低 30\% $40 \%{ }^{[18]}$. 水面蒸发与蒸发皿蒸发之间的差 异是由蒸发血. 本身结构所引起的 ${ }^{[48]}$, 受到 
蒸发血容积的限制, 蒸发血的水量和水深远远小于实际湖泊水体, 其具有较小的热储存能力, 更容易受到季 节和环境的影响 ${ }^{[49]}$.

\section{3 环境因子影响机制}

鄱阳湖夏季水面日蒸发量和蒸发血目 蒸发量与环境因子 (净辐射 Rn、气温 $\mathrm{Ta}$ 、风 速、饱和水汽压差 VPD、相对湿度 RH、水温 $\mathrm{Tw}$ 和气温日较差 DT) 的相关分析如表 1 所 示. 水面日蒸发量与风速的相关性最显著 $(r=0.506$, 通过 99\% 置信区间检验). 其次 是相对湿度 $(r=-0.477)$ 和水温 $(r=$ $0.456)$, 而与净辐射和气温无显著相关性. 蒸发血蒸发量与净辐射 $(r=0.569)$, 饱和水 汽压差 $(r=0.520)$ 、相对湿度 $(r=-0.499)$ 和气温 $(r=0.424)$ 均显著相关, 通过 $99 \%$ 置 信区间检验. 而与其他因子无显著相关性 (表 1 ).

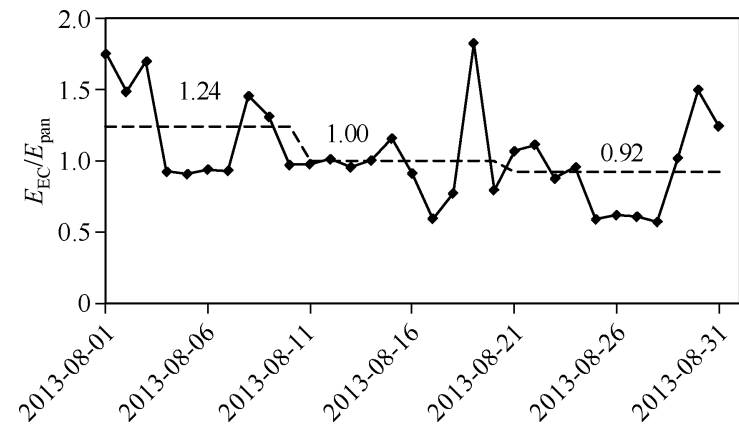

图 6 鄱阳湖 2013 年 8 月份实际水面日蒸发量 $\left(E_{\mathrm{EC}}\right)$

与蒸发血日蒸发量 $\left(E_{\mathrm{pan}}\right)$ 的比值 $\left(E_{\mathrm{EC}} / E_{\mathrm{pan}}\right)$ 变化

Fig. 6 Ratio of water evaporation to pan

evaporation over the Lake Poyang in August, 2013

通过逐步回归方法, 得到水面日蒸发量与环境因子相关关系的回归方程: $E_{\mathrm{EC}}=0.35 \mathrm{WS}+0.60 \mathrm{Tw}-$ $0.53 \mathrm{DT}-0.097 \mathrm{RH}-4.54\left(R^{2}=0.628, R M S E=1.44 \mathrm{~mm} / \mathrm{d}\right)$, 风速、水温、相对湿度和气温日较差通过检验进 人回归方程. 在日尺度上, 方程能解释潜热通量变化的 $63 \%$. 蒸发血蒸发量的回归方程为 $E_{\mathrm{pan}}=0.018 \mathrm{Rn}+$ $3.193\left(R^{2}=0.324, R M S E=0.99 \mathrm{~mm} / \mathrm{d}\right)$, 尽管在单因子分析时有多个因子与蒸发血蒸发量显著相关, 但仅有 净辐射通过检验进人回归方程, 方程仅能解释蒸发血蒸发量变化的 $32 \%$. 即使考虑气温、饱和水汽压差、相 对湿度和气温日较差, 回归方程的相关系数仅提高到 0.40 .

通过单因子相关分析发现,鄱阳湖夏季水面蒸发速率和蒸发血蒸发速率与环境因子的关系不尽相同. 蒸发血蒸发速率与气温、净辐射呈很好的正相关关系 $\left(R^{2}\right.$ 分别为 0.18 和 0.32 ), 而水面蒸发速率与其相关性 则不明显. 两者与 VPD 均呈明显的正相关关系. 水面蒸发速率与风速呈显著正相关, 与气温日较差呈负相 关, 而蒸发血蒸发速率与两者无显著相关性 (表 1). 水面蒸发受到动力因素 (风速等) 和热力因素 (气温和净 辐射等) 综合作用的影响 ${ }^{[5]}$. 任国玉等 ${ }^{[30]}$ 研究表明, 蒸发量主要受到日照时数、平均风速和温度日较差的影 响. 并通过这些因素呈现的减少趋势, 来解释我国蒸发血蒸发量近 50 年来长期趋向减少现象. 鄱阳湖夏季 水面蒸发并未表现出与气温和辐射等热力因素显著相关, 这是因为 8 月份气温和净辐射等变化幅度不大, 因此表现为风速等动力因素是水面蒸发的主要控制因子. 而与大水面的水面蒸发相比, 位于棠荫岛上的蒸 发血观测更容易受到周围环境的影响. Venäläinen 等 ${ }^{[50]}$ 认为水面蒸发受水体面积影响. 由于蒸发血水体容 积小, 与湖泊相比水体热容量较小, 因此与大气热交换更加强烈 ${ }^{[17]}$.

\section{3 结论}

水面蒸发研究对于区域气候、旱涝变化趋势, 水资源形成及变化规律,水资源评价等具有重要的意义. 本研究基于浴度相关系统观测鄱阳湖夏季水面蒸发, 分析了水面蒸发在小时和日尺度的变化规律, 对比分 析了夏季水面蒸发与蒸发血蒸发在变化趋势和对环境因子响应方面的差异. 水面蒸发日变化波动剧烈, 无 明显的变化规律. 其变化过程与净辐射无明显相关性, 而主要受风速的影响. 说明在日尺度上, 水面蒸发主 要受动力驱动. 鄱阳湖 8 月份日水面蒸发量与蒸发血蒸发量具有很好的一致性, 均呈下降的趋势. 但其比值 变化较大, 表现为 8 月初高值时, 蒸发血蒸发低估实际水面蒸发, 而在月末低值时, 高估实际水面蒸发. 与环 境因子的相关分析发现, 鄱阳湖夏季日水面蒸发量主要受风速和相对湿度的影响, 而蒸发血蒸发受净辐射、 气温、饱和水汽压差等因素的影响. 湖泊水体蒸发对于了解湖泊水量变化及区域水循环和水资源具有重要 意义. 
表 1 鄱阳湖 2013 年 8 月份水面日蒸发速率和蒸发血日蒸发速率与环境因子的相关关系 (样本数为 31 )

Tab. 1 The Pearson correlation analysis within daily water evaporation, pan evaporation and controlling factors over the Lake Poyang in August, 2013

( The number of sample is 31 )

\begin{tabular}{llllcccr}
\hline & 净辐射 & 气温 & 风速 & 饱和水汽压差 & 相对湿度 & 水温 & 气温日较差 \\
\hline 水面蒸发速率 & 0.077 & 0.127 & $0.506^{* *}$ & $0.396^{*}$ & $-0.477^{* *}$ & $0.456^{*}$ & -0.336 \\
蒸发而蒸发速率 & $0.569^{* *}$ & $0.424^{* *}$ & 0.051 & $0.520^{* *}$ & $-0.499^{* *}$ & 0.308 & 0.232 \\
\hline
\end{tabular}

* 表示通过 $95 \%$ 置信区间检验; $* *$ 表示通过 $99 \%$ 置信区间检验.

致谢: 感谢鄱阳湖水文局提供蛇山岛水文数据. 感谢蛇山岛基地工作人员在野外仪器维护等给予的帮助. 感 谢江西省水文局洪全祥处长、江西省鄱阳湖水文局龚向民副调研员、中国科学院南京地理与湖泊研究所冯 徽徽博士等对仪器安装和维护给予的帮助.

\section{4 参考文献}

[ 1 ] Huntington TG. Evidence for intensification of the global water cycle: review and synthesis. Journal of Hydrology, 2006, 319 (1) : $83-95$.

[ 2 ] 裴步祥. 蒸发和蒸散的测定与计算. 北京:气象出版社,1989.

[ 3 ] Grismer M, Orang M, Snyder R et al. Pan evaporation to reference evapotranspiration conversion methods. Journal of Irrigation and Drainage Engineering, 2002, 128(3):180-184.

[ 4 ] 毛 锐,高俊峰. 太湖地区湖泊水面蒸发. 北京:科学技术文献出版社, 1993.

[ 5 ] Brutsaert W, Parlange M. Hydrologic cycle explains the evaporation paradox. Nature, 1998, 396(6706) :30.

[ 6 ] Ohmura A, Wild M. Is the hydrological cycle accelerating? Science, 2002, 298 (5597) :1345-1346.

[ 7 ] Baldocchi DD. Assessing the eddy covariance technique for evaluating carbon dioxide exchange rates of ecosystems: past, present and future. Global Change Biology, 2003, 9(4):479-492.

[ 8 ] 盛 琼, 申双和,顾 泽. 小型蒸发器的水面蒸发量折算系数. 南京气象学院学报, 2007, 30(4):561-565.

[ 9 ] Sene K, Gash J, McNeil D. Evaporation from a tropical lake: comparison of theory with direct measurements. Journal of Hydrology, 1991, 127 ( 1 ) : 193-217.

[10] Stannard DI, Rosenberry DO. A comparison of short-term measurements of lake evaporation using eddy correlation and energy budget methods. Journal of Hydrology, 1991, 122(1) :15-22.

[11] Blanken PD, Rouse WR, Culf AD et al. Eddy covariance measurements of evaporation from Great Slave Lake, Northwest Territories, Canada. Water Resources Research, 2000, 36(4):1069-1077.

[12] Rouse WR, Oswald CJ, Binyamin J et al. The role of northern lakes in a regional energy balance. Journal of Hydrometeorology, 2005, 6(3):291-305.

[13 ] Rouse WR, Blanken PD, Bussières N et al. An investigation of the thermal and energy balance regimes of Great Slave and Great Bear Lakes. Journal of Hydrometeorology, 2008, 9(6):1318-1333.

[14] Liu, H, Zhang Y, Liu S et al. Eddy covariance measurements of surface energy budget and evaporation in a cool season over southern open water in Mississippi. Journal of Geophysical Research, 2009, 114(D4) : D04110.

[15] Anderson DE, Striegl RG, Stannard DI et al. Estimating lake-atmosphere $\mathrm{CO}_{2}$ exchange. Limnology and Oceanography, 1999, 44 :988-1001.

[16] Assouline S, Tyler SW, Tanny J et al. Evaporation from three water bodies of different sizes and climates: Measurements and scaling analysis. Advances in Water Resources, 2008, 31(1):160-172.

[17] Nordbo A, Launiainen S, Mammarella I et al. Long-term energy flux measurements and energy balance over a small boreal lake using eddy covariance technique. Journal of Geophysical Research, 2011, 116(D2) :D02119. 1-D02119.17.

[18 ] Lenters JD, Kratz TK, Bowser CJ. Effects of climate variability on lake evaporation: Results from a long-term energy budget study of Sparkling Lake, northern Wisconsin( USA). Journal of Hydrology, 2005, 308(1) : 168-195.

[19] 闵 寒. 水面蒸发器折算系数昼夜差别初步分析. 水文, 1988,4:14.

[20] 施成熙, 牛克源, 陈天珠等. 水面蒸发器折算系数研究. 地理科学, 1986,6 (4) :305-313. 
[21] 牛振红,孙 明. 水面蒸发折算系数的对比观测实验与分析计算. 水文,2003,23(3): 49-51.

[22］王远明,李成荣. 宜昌站水面蒸发折算系数分析. 人民长江, 1999,30(1) :41-45.

[23] 王 梅,王建波,那景坤. E-601 型蒸发器水面蒸发实验分析. 黑龙江水专学报, 2004,31 (3) : 10-12.

[24] 毛 锐. 太湖水面蒸发量预报模型及其应用. 湖泊科学, 1992,4(4):8-13.

[25]施成熙,市毓明,朱晓原. 确定水面蒸发模型. 地理科学, 1984,4 (1) :1-10.

[26] 渓培民. 水面蒸发与散热系数公式研究 (一). 湖泊科学, 1994,6(1):1-12.

[27] 赵振国. 水面蒸发系数公式探讨. 水利学报, 2009,40(12): 1440-1443.

[28］李万义. 适用于全国范围的水面蒸发量计算模型的研究. 水文, 2000,20(4) : 13-17.

[29] 王永义,水面蒸发计算方法及其检验. 地下水,2006. 28(2) : 15-16.

[30］任国玉,郭 军. 中国水面蒸发量的变化. 自然资源学报,2006,21(1):31-44.

[31] 闵 骞. 鄱阳湖水面蒸发规律初探. 水文, 1994,6:35-42.

[32] 闵骞,苏宗萍,王叙军. 近 50 年鄱阳湖水面蒸发变化特征及原因分析. 气象与减灾研究, 2007, 30(3): 17-20.

[33] 王艳君,姜 䑣,许崇育. 长江流域蒸发血蒸发量及影响因素变化趋势. 自然资源学报,2005,20(6) :864-870.

[34] 闵 骞, 刘 影. 鄱阳湖水面蒸发量的计算与变化趋势分析 (1955-2004 年). 湖泊科学, 2006,18 (5):452-457.

[35] 肖 薇, 刘寿东, 李旭辉等. 大型浅水湖泊与大气之间的动量和水热交换系数一一太湖为例. 湖泊科学, 2012,24 (6) :932-942.

[36] 谢冬明,郑 鹏,邓红兵等. 鄱阳湖湿地水位变化的景观响应. 生态学报,2011,31(5):1269-1276.

[37] Wilczak JM, Oncley S, Stage SA. Sonic anemometer tilt correction algorithms. Boundary-Layer Meteorology, 2001, 99 (1) $: 127-150$.

[38 ] Moore C. Frequency response corrections for eddy correlation systems. Boundary-Layer Meteorology, 1986, 37(1/2) :1735.

[39] Van Dijk AI. Estimates of $\mathrm{CO}_{2}$ uptake and release among European forests based on eddy covariance data. Global Change Biology, 2004, 10(9) :1445-1459.

[40 ] Webb EK, Pearman GI, Leuning R. Correction of flux measurements for density effects due to heat and water vapour transfer. Quarterly Journal of the Royal Meteorological Society, 1980, 106(447) :85-100.

[41] Göckede M, Foken T, Aubinet M et al. Quality control of CarboEurope flux data-Part 1: Coupling footprint analyses with flux data quality assessment to evaluate sites in forest ecosystems. Biogeosciences, 2008, 5(2):433-450.

[42] Foken T, Göockede M, Mauder M et al. Post-field data quality control, in Handbook of micrometeorology. Dordrecht: Springer, $2005: 181-208$.

[43] Falge E, Baldocchi D, Olson R et al. Gap filling strategies for defensible annual sums of net ecosystem exchange. Agricultural and Forest Meteorology, 2001, 107(1):43-69.

[44] Hsieh CI, Katul G, Chi T. An approximate analytical model for footprint estimation of scalar fluxes in thermally stratified atmospheric flows. Advances in Water Resources, 2000, 23(7) :765-772.

[45] Haenel HD, Grünhage L. Footprint analysis: a closed analytical solution based on height-dependent profiles of wind speed and eddy viscosity. Boundary-Layer Meteorology, 1999, 93(3):395-409.

[46] Schmid HP. Footprint modeling for vegetation atmosphere exchange studies: a review and perspective. Agricultural and Forest Meteorology, 2002, 113(1):159-183.

[47 ] Tanny J, Cohen S, Assouline S et al. Evaporation from a small water reservoir: Direct measurements and estimates. Journal of Hydrology, 2008, 351(1):218-229.

[48] Stauffer RE. Testing lake energy budget models under varying atmospheric stability conditions. Journal of Hydrology, $1991, \mathbf{1 2 8}(1): 115-135$.

[49] Krabbenhoft DP, Bowser CJ, Anderson MP et al. Estimating groundwater exchange with lakes: 1 . The stable isotope mass balance method. Water Resources Research, 1990, 26(10):2445-2453.

[50] Venäläinen A, Frech M, Heikinheimo M et al. Comparison of latent and sensible heat fluxes over boreal lakes with concurrent fluxes over a forest: Implications for regional averaging. Agricultural and Forest Meteorology, 1999. 98:535-546. 